University of Nebraska - Lincoln

DigitalCommons@University of Nebraska - Lincoln

\title{
The effective magnetoelectroelastic moduli of matrix-based multiferroic composites
}

\author{
Shashidhar Srinivas \\ University of Nebraska - Lincoln \\ Jiangyu Li \\ University of Nebraska-Lincoln, jjli@uw.edu
}

Y.C. Zhou

Key Laboratory of Advanced Materials and Rheological Properties of Ministry of Education, Faculty of Materials and Optoelectronic Physics, Xiangtan University, China, Xiangtan, Hunan 41105, China

A.K. Soh

University of Hong Kong, China

Follow this and additional works at: https://digitalcommons.unl.edu/cmrafacpub

Part of the Nanoscience and Nanotechnology Commons

Srinivas, Shashidhar; Li, Jiangyu; Zhou, Y.C.; and Soh, A.K., "The effective magnetoelectroelastic moduli of matrix-based multiferroic composites" (2006). Faculty Publications from Nebraska Center for Materials and Nanoscience. 14.

https://digitalcommons.unl.edu/cmrafacpub/14

This Article is brought to you for free and open access by the Materials and Nanoscience, Nebraska Center for (NCMN) at DigitalCommons@University of Nebraska - Lincoln. It has been accepted for inclusion in Faculty Publications from Nebraska Center for Materials and Nanoscience by an authorized administrator of DigitalCommons@University of Nebraska - Lincoln. 


\title{
The effective magnetoelectroelastic moduli of matrix-based multiferroic composites
}

\author{
Shashidhar Srinivas and J. Y. Li ${ }^{\mathrm{a})}$ \\ Department of Engineering Mechanics and Center for Materials Research and Analysis, \\ University of Nebraska-Lincoln, Lincoln, Nebraska 68588-0526 \\ Y. C. Zhou \\ Key Laboratory of Advanced Materials and Rheological Properties of Ministry of Education, \\ Faculty of Materials and Optoelectronic Physics, Xiangtan University, China, Xiangtan, \\ Hunan 41105, China
}

A. K. Soh

Department of Mechanical Engineering, University of Hong Kong, China

(Received 16 August 2005; accepted 21 December 2005; published online 24 February 2006)

In this paper, we develop a mean field Mori-Tanaka model [T. Mori and K. Tanaka, Acta. Metall. 21, 571 (1973)] to calculate the effective magnetoelectroelastic moduli of matrix-based multiferroic composites, emphasizing the effects of shape and orientation distribution of second phase particles that have not been investigated before. Through a systematic study, it is observed that laminated composites are optimal for magnetoelectric coefficient $a_{11}$, while fibrous composites are optimal for $a_{33}$. In addition, these coupling coefficients are maximum when the second phase particles are aligned. It is also postulated that the large discrepancy between theoretical predictions and experimental measurements for magnetoelectric coefficients of multiferroic composites previously reported is partly due to the orientation distribution of second phase particles, which has not been considered before in theoretical modeling. When our calculations take the orientation distribution of second phase particles into account with appropriate texture coefficient, good agreement with experimental data is observed. (C) 2006 American Institute of Physics. [DOI: 10.1063/1.2173035]

\section{INTRODUCTION}

Multiferroic materials possess two or more types of orders simultaneously. For example, ferroelectromagnets display both magnetic and electric orderings, and thus allow the interconversion of magnetic and electric energies. There is great interest in magnetoelectric materials that generate an electrical response by an external magnetic field, or vice versa. Applications of such materials include magnetoelectric sensors in optoelectronics and microwave electronics. The magnetoelectric and piezomagnetic couplings were initially thought to be forbidden by the symmetry requirement because time reversal changes the sign of magnetic field $\mathbf{H}$ while leaving electric field $\mathbf{E}$ invariant, until Landau and Lifshitz pointed out that such effects can exist in materials with a magnetic structure. ${ }^{1,2}$ The observation of magnetoelectric effect was reported by $\mathrm{Astrov}^{3}$ in a single crystal of antiferromagnetic $\mathrm{Cr}_{2} \mathrm{O}_{3}$, in which magnetoelectric effect induced by both electric and magnetic fields were observed. ${ }^{4,5}$ Subsequently observations of magnetoelectric effect in more crystals have been reported, including $\mathrm{BiFeO}_{3}$ (Ref. 6) and $\mathrm{YMnO}_{3}{ }^{7}$ However, the magnetoelectric effect in those single-phase materials is usually very small, and there is great effort devoted in developing multiferroic composites that possess large magnetoelectric coupling coefficients.

\footnotetext{
a) Author to whom correspondence should be addressed; present address: Department of Mechanical Engineering, University of Washington, Seattle, WA 98195-2600; Fax: 206-685-8047; electronic mail: jjli@u.washington.edu
}

The multiferroic composite was reported in $1970 \mathrm{~s},{ }^{8}$ consisting of a piezoelectric phase $\mathrm{BaTiO}_{3}$ and a ferromagnetic phase $\mathrm{CoFe}_{2} \mathrm{O}_{4}$. Since then, a variety of multiferroic composites have been fabricated, with piezoelectric phase includes $\mathrm{BaTiO}_{3}$, lead zirconic titanate (PZT), and polyvinylidene fluoride (PVDF), and ferromagnetic phase includes $\mathrm{CoFe}_{2} \mathrm{O}_{4}$, nickel ferrite, and $\mathrm{Tb}_{1-x} \mathrm{Dy}_{x} \mathrm{Fe}_{2} \cdot{ }^{9-20}$ Recently, a self-assembled multiferroic nanocomposite has also been reported, ${ }^{21}$ with hexagonal arrays of $\mathrm{CoFe}_{2} \mathrm{O}_{4}$ nanopillars embedded in a $\mathrm{BaTiO}_{3}$ matrix. Despite these progresses, the experimentally observed magnetoelectric coefficients in most of those composites are usually much smaller than the theoretical predictions. ${ }^{10,11}$ On one hand, this suggests that there is much room for improvement in materials processing. On the other hand, it also calls for more reliable theoretical models that approximate the composite microstructures better. For example, in a multiferroic composites consisting of a matrix and a second phase, it is usually very difficult to align all the particles of the second phase during processing, making the effect of the orientations and textures of particles important. Such effect has rarely been investigated. In addition, most composites investigated so far, experimentally or theoretically, are either laminated or fibrous in microgeometry. However, it is not clear whether such microstructure is optimal or not, as far as the magnetoelectric coupling is concerned. We intend to address these issues in this paper.

A variety of models have been proposed in the last 10 years to predict the effective magnetoelectroelastic moduli of multiferroic composites. For example, Nan ${ }^{22}$ used Green's 
function method combined with perturbation theory to study a fibrous composites consisting of $\mathrm{CoFe}_{2} \mathrm{O}_{4}$ and $\mathrm{BaTiO}_{3}$. For such fibrous composites, exact connections among effective magnetoelectroelastic moduli were derived by Benveniste using a uniform field concept. ${ }^{23}$ Particulate composite was investigated by Harshe et al. using a cubic model, ${ }^{10}$ and multilayered laminate was studied by Avellaneda and Harshe. ${ }^{24}$ A mean field Mori-Tanaka model has been generalized to multiferroic composites by $\mathrm{Li}$ and Dunn, ${ }^{25}$ among others, and frequency dependence of magnetoelectric coefficients of multiferroic laminates were modeled by Bichurin et al. ${ }^{26,27}$ Recently, the polycrystalline multiferroic composites have also been investigated by Srinivas and Li using a selfconsistent approach. ${ }^{28}$ Despite these progresses, most of the studies concentrated on either fibrous composites or laminated composites, without addressing the effect of shape of second phase. In addition, most of studies concerned matrixbased composites with aligned second phase, without addressing the effect of orientations and texture of particles. The only exception is a recent article by Shi et al. that studied the effect of orientation of piezoelectric particles using a cutoff angle. ${ }^{29}$ These simplified microgeometries to some extent caused the discrepancy between theoretical predictions and experimental measurements reported earlier. ${ }^{10,11}$ We intend to address those deficiencies in this paper. In particular, we will use Mori-Tanaka model, ${ }^{30-32}$ combined with orientational averaging, to study the effects of shape, orientation, texture, and volume fraction of second phase particles on the overall responses of the composites, with the objective to reliably predict the effective magnetoelectric coefficients of the composites and identify the optimal shape and textures of the second phase particles for the magnetoelectric coupling.

The paper is organized as follows. The basic equations and notations regarding the magnetoelectroelasticity are given in Sec. II, and a micromechanical model for matrixbased multiferroic composites is derived in Sec. III, where the equations regarding the particles' shape and orientation distribution are presented systematically. Numerical results and discussions regarding the effects of particles' shape and orientation distribution will then be presented in Sec. IV, where good agreement between theoretical predictions and experimental measurements is observed when appropriate texture coefficient is adopted.

\section{MAGNETOELECTROELASTICITY}

We consider the linear magnetoelectroelastic effect, where the static magnetic, electric, and elastic fields are coupled through the following constitutive equations:

$$
\begin{aligned}
& \sigma_{i j}=C_{i j k l} \varepsilon_{k l}+e_{i j l}\left(-E_{l}\right)+q_{i j l}\left(-H_{l}\right), \\
& D_{i}=e_{i k l} \varepsilon_{k l}-\kappa_{i l}\left(-E_{l}\right)-a_{i l}\left(-H_{l}\right), \\
& B_{i}=q_{i k l} \varepsilon_{k l}-a_{i l}\left(-E_{l}\right)-\mu_{i l}\left(-H_{l}\right) .
\end{aligned}
$$

Here $\sigma_{i j}$ and $\varepsilon_{i j}$ are the elastic stress and strain, $D_{i}$ and $E_{i}$ are the electric displacement and field, and $B_{i}$ and $H_{i}$ are the magnetic intensity and field. $C_{i j k l}, \kappa_{i l}$, and $\mu_{i l}$ are the elastic stiffness, the dielectric, and magnetic permeability tensors, respectively. These tensors directly connect like fields, e.g., stresses to strains. Elastic field is coupled to the electric and magnetic fields through the piezoelectric coefficient $e_{i j l}$ and piezomagnetic coefficient $q_{i j l}$, respectively, while electric and magnetic fields are coupled through the magnetoelectric coefficient $a_{i l}$. In the constitutive equations, $-E_{i}$ and $-H_{i}$ are used instead of $E_{i}$ and $H_{i}$, as they enable the construction of a symmetric matrix of constitutive moduli.

The strain, electric, and magnetic fields can be derived from gradient equations,

$$
\begin{aligned}
& \varepsilon_{i j}=\frac{1}{2}\left(u_{i, j}+u_{j, i}\right), \\
& E_{i}=-\phi_{, i}, \\
& H_{i}=-\psi_{, i},
\end{aligned}
$$

where $u_{i}$ is the elastic displacement, $\phi$ and $\psi$ are the electric and magnetic potentials, respectively, and the subscript comma is used to denote partial differentiation with respect to $x_{i}$. On the other hand, when there are no free charge and body force assumed to exist, the stress, electric displacement, and magnetic intensity satisfy the following equilibrium equations:

$$
\begin{aligned}
& \sigma_{i j, i}=0, \\
& D_{i, i}=0, \\
& B_{i, i}=0,
\end{aligned}
$$

where we have used summation convection for repeated subscript.

The constitutive, gradient, and equilibrium equations can be rewritten in a more concise form as follows, generalizing a notation introduced by Barnett and Lothe, ${ }^{33}$

$$
\sum_{i J}=L_{i J K l} Z_{K l}
$$

$$
Z_{K l}=U_{K, l}
$$

$$
\sum_{i J, i}=0
$$

with

$$
\sum_{i J}=\left\{\begin{array}{l}
\sigma_{i j} \\
D_{i} \\
B_{i}
\end{array}, \quad Z_{J i}=\left\{\begin{array}{ll}
\varepsilon_{i j} \\
-E_{i} \\
-H_{i}
\end{array} \quad, \quad U_{J}= \begin{cases}u_{j}, & J=1,2,3 \\
\phi, & J=4 \\
\psi, & J=5,\end{cases}\right.\right.
$$

and 


$$
L_{i J K l}=\left\{\begin{array}{lll}
C_{i j k l}, & J, K=1,2,3 \\
e_{i j l}, & K=4, & J=1,2,3 \\
q_{i j l}, & K=5, & J=1,2,3 \\
e_{i k l}, & J=4, & K=1,2,3 \\
-\kappa_{i l}, & J=4, & K=4 \\
-a_{i l}, & J=4, & K=5 \\
q_{i k l}, & J=5, & K=1,2,3 \\
-a_{i l}, & J=5, & K=4 \\
-\mu_{i l}, & J=5, & K=5,
\end{array}\right.
$$

where both upper and lower case subscripts are used. The upper case subscript ranges from 1 to 5 and the lower case subscript ranges from 1 to 3 . Repeated upper case subscripts are summed from 1 to 5 . When the standard matrix notation for tensors ${ }^{34}$ is adopted, the constitutive equation can be written as

$$
\Sigma=\mathbf{L Z}
$$

with

$$
\boldsymbol{\Sigma}=\left[\begin{array}{l}
\sigma \\
\mathbf{D} \\
\mathbf{B}
\end{array}\right], \quad \mathbf{Z}=\left[\begin{array}{c}
\boldsymbol{E} \\
-\mathbf{E} \\
-\mathbf{H}
\end{array}\right], \quad \mathbf{L}=\left[\begin{array}{ccc}
\mathbf{C} & \mathbf{e} & \mathbf{q} \\
\mathbf{e}^{\mathrm{t}} & -\boldsymbol{\kappa} & -\mathbf{a} \\
\mathbf{q}^{\mathrm{t}} & -\mathbf{a}^{\mathrm{t}} & -\boldsymbol{\mu}
\end{array}\right],
$$

where the superscript $t$ is used to denote the matrix transpose.

\section{MULTIFERROIC COMPOSITES}

\section{A. The effective moduli}

We now consider a matrix-based multiphase multiferroic composite, with the constitutive equation of phase $r$ given by

$$
\boldsymbol{\Sigma}_{\mathrm{r}}=\mathbf{L}_{\mathrm{r}} \mathbf{Z}_{\mathrm{r}}
$$

where phase 1 denotes matrix. We are interested in determining the macroscopic properties of the multiferroic composites in terms of their microstructures, and propose that the behavior of the multiferroic composites with macroscopic homogeneity is governed by the effective constitutive equation,

$$
\overline{\mathbf{\Sigma}}=\mathbf{L}^{*} \overline{\mathbf{Z}},
$$

where $\mathbf{L}^{*}$ is the effective magnetoelectroelastic moduli of the composite, and the overhead bar is used to denote the volume averaging. Due to the linearity, we have

$$
\mathbf{Z}_{r}=\mathbf{A}_{r} \overline{\mathbf{Z}},
$$

where $\mathbf{A}_{r}$ is the concentration factor of phase $r$, satisfying

$$
\overline{\mathbf{A}}=\mathbf{I},
$$

and $\mathbf{I}$ is a $9 \times 9$ unit matrix. As a result, we have

$$
\mathbf{L}^{*}=\sum_{r=1}^{N} f_{r} \mathbf{L}_{r} \mathbf{A}_{r},
$$

from which the effective moduli can be determined for a $\mathrm{N}$-phase composite, if the concentration factor is known. In the equation $f_{r}$ is the volume fraction of phase $r$.
In order to determine the effective magnetoelectroelastic moduli of multiferroic composite, aproximation must be made regarding the distribution of magnetoelectroelastic field in the composite. We turn to a micromechanical model for this purpose. For elastic or piezoelectric composites, the Mori-Tanaka model is very powerful in predicting the effective elastic, piezoelectric, and dielectric moduli of the composites ${ }^{30,35}$ which is essentially a mean field method, ${ }^{31,32}$ and usually leads to the effective constitutive moduli that lie inside the Hashin-Shtrikman bounds. ${ }^{36}$ Using this model, the concentration factor of phase $r$ can be determined as

$$
\mathbf{A}_{r}=\mathbf{A}_{r}^{\mathrm{dil}}\left(\sum_{i=1}^{N} f_{i} \mathbf{A}_{i}^{\mathrm{dil}}\right)^{-1},
$$

with the dilute concentration factor $\mathbf{A}_{r}^{\text {dil }}$ given by

$$
\mathbf{A}_{r}^{\text {dil }}=\left[\mathbf{I}+\mathbf{S}_{r} \mathbf{L}_{1}^{-1}\left(\mathbf{L}_{r}-\mathbf{L}_{1}\right)\right]^{-1},
$$

where $\mathbf{S}_{r}$ is the magnetoelectroelastic Eshelby tensor ${ }^{37,38}$ for phase $r$, which is a function of the magnetoelectroelastic moduli of matrix, and the shape and orientation of phase $r$, and superscript -1 is used to denote the matrix inverse. The essence of Mori-Tanaka model is that the averaging field in phase $r$ of the composite is equivalent to the field in a single particle embedded in an infinite medium, with the unknown averaging field in the composite matrix applied at the boundary. It is well known that when the particle shape is ellipsoidal, the field within the single particle is uniform, which can be evaluated using Eshelby tensor. For fibrous or penny shape particle, the closed form expressions for magnetoelectroelastic Eshelby tensor were derived by Li and Dunn. ${ }^{38}$ For more general shapes, a numerical algorithm for the evaluation of Eshelby tensor was given by $\mathrm{Li}^{39}$

Our model clearly demonstrates how the magnetoelectric effect is induced in a piezoelectric-piezomagnetic composite. For example, when a piezomagnetic particle is embedded in a piezoelectric matrix subjected to an applied electric field, a magnetic induction will be induced in the piezomagnetic particle due to the electromechanical interaction in piezoelectric phase and magnetomechanical interaction in piezomagnetic phase, as shown in Eq. (14). It is also emphasized that our current model has the following advantages compared with previous studies. Firstly, it allows us to study the effect of shape of the second phase particles. In particular, the particles are assumed to be spheroidal, with $\alpha=A_{3} / A_{1}=A_{3} / A_{2}$ which is continuously varying from 0 to $\infty$, where $A_{i}$ is the dimensions of spheroids. Combining it with our numerical algorithm to evaluate Eshelby's tensor for general ellipsoids, we are able to study a wide range of particle shapes of second phase, with $\alpha \rightarrow \infty$ for fibrous particles, $\alpha=1$ for spherical particles, and $\alpha \rightarrow 0$ for lamellar particles. Most of previous studies focused on a particular microgeometry such as laminated or fibrous composites. Secondly, the multiphase treatment also allows us to study the effect of orientation distribution of second phase particles, by regarding particles at different orientations as different phases. Most previous studies concerned two-phase composites or at most threephase composites, and rarely addressed the effect of par- 
ticles' orientation. As we show later, the effects of particles shape and orientation distribution are very important.

\section{B. Two-phase composites with particles of varying orientations}

To be specific we focus on a two-phase composite consisting of a matrix and a second phase. The particles of the second phase have identical shape, but varying orientations. As such, two different kinds of coordinate systems can be established, one is global fixed on the matrix, and the other is local fixed on particles of different orientations. The orientations of different particles can then be described by Euler angles $(\theta, \psi, \phi) .^{40}$ Due to the anisotropy of constitutive moduli, particles of different orientations will have different constitutive moduli in a global coordinate system, and thus orientational averaging has to be carried out over particles at all orientations first. As a result, the effective constitutive moduli are given by

$$
\mathbf{L}^{*}=f_{1} \mathbf{L}_{1} \mathbf{A}_{1}+f_{2}\left\langle\mathbf{L}_{2}(\theta, \psi, \phi) \mathbf{A}_{2}(\theta, \psi, \phi)\right\rangle,
$$

with $\langle\cdots\rangle$ used to denote the orientational averaging,

$$
\begin{aligned}
\langle H(\theta, \psi, \phi)\rangle= & \int_{0}^{2 \pi} \int_{0}^{2 \pi} \int_{0}^{\pi} H(\theta, \psi, \phi) W(\theta, \psi, \phi) \\
& \times \sin \theta d \theta d \psi d \phi,
\end{aligned}
$$

where $W(\theta, \psi, \phi)$ is the orientation distribution function
(ODF), which gives the probability of locating a particle at orientation $(\theta, \psi, \phi)$. When the Mori-Tanaka model is adopted, the concentration factor is evaluated from

$$
\mathbf{A}_{2}(\theta, \psi, \phi)=\mathbf{A}_{2}^{\operatorname{dil}}(\theta, \psi, \phi)\left[f_{1} \mathbf{I}+f_{2}\left\langle\mathbf{A}_{2}^{\operatorname{dil}}(\theta, \psi, \phi)\right\rangle\right]^{-1},
$$

and

$$
\mathbf{A}_{1}=\left[f_{1} \mathbf{I}+f_{2}\left\langle\mathbf{A}_{2}^{\text {dil }}(\theta, \psi, \phi)\right\rangle\right]^{-1}
$$

with

$$
\mathbf{A}_{2}^{\operatorname{dil}}(\theta, \psi, \phi)=\left\{\mathbf{I}+\mathbf{S}(\theta, \psi, \phi) \mathbf{L}_{1}^{-1}\left[\mathbf{L}_{2}(\theta, \psi, \phi)-\mathbf{L}_{1}\right]\right\}^{-1} .
$$

In those equations, $\mathbf{L}(\theta, \psi, \phi)$ can be obtained from their principle values using transformation rules for second, third, and fourth rank tensors,

$$
\begin{aligned}
& a_{i j}(\theta, \psi, \phi)=T_{i k} T_{j l} a_{k l}, \\
& e_{i j k}(\theta, \psi, \phi)=T_{i l} T_{j m} T_{k n} e_{l m n}, \\
& C_{i j k l}(\theta, \psi, \phi)=T_{i m} T_{j n} T_{k o} T_{l p} C_{m n o p},
\end{aligned}
$$

where the transformation matrix is given by

$$
\mathbf{T}^{-1}=\left[\begin{array}{ccc}
\cos \psi \cos \theta \cos \phi-\sin \psi \sin \phi & \sin \psi \cos \theta \cos \phi+\cos \psi \sin \phi & -\sin \theta \cos \phi \\
-\cos \psi \cos \theta \sin \phi-\sin \psi \cos \phi & -\sin \phi \cos \theta \sin \phi+\cos \psi \cos \phi & \sin \theta \sin \phi \\
\cos \psi \sin \theta & \sin \psi \sin \theta & \cos \theta
\end{array}\right]
$$

In this study, we adopt Gaussian distribution function for ODF,

$$
W(\theta, \psi, \phi)=\frac{1}{\mu \sqrt{2 \pi}} e^{-\left(\theta^{2} / 2 \mu^{2}\right)},
$$

which can be used to approximate a wide range of textures by varying $\mu$. For example, the random orientation distribution of particles, where $W(\theta, \psi, \phi)=1$, can be obtained by letting $\mu \rightarrow \infty$, while the aligned distribution of particles, where $W(\theta, \psi, \phi)=\delta(\theta)$, can be obtained by letting $\mu \rightarrow 0$. Thus $\mu$ can be adjusted for different ODFs and will be called orientation distribution coefficient or texture coefficient here. In addition, the orientational averaging is difficult to evaluate analytically in general, and we adopt Gaussian quadrature method for numerical integration, in which the integration is approximated by the sum of the value of its integrand at a set of points called abscissa, multiplied by weighting coefficient $w_{i j k}$,
TABLE I. Material properties of $\mathrm{BaTiO}_{3}$ and $\mathrm{CoFe}_{2} \mathrm{O}_{4}$

\begin{tabular}{lcc}
\hline \hline & $\mathrm{BaTiO}_{3}$ & $\mathrm{CoFe}_{2} \mathrm{O}_{4}$ \\
\hline$C_{11}(\mathrm{GPa})$ & 166 & 286 \\
$C_{12}(\mathrm{GPa})$ & 77 & 173 \\
$C_{13}(\mathrm{GPa})$ & 78 & 170 \\
$C_{33}(\mathrm{GPa})$ & 162 & 269.5 \\
$C_{44}(\mathrm{GPa})$ & 43 & 45.3 \\
$e_{15}\left(\mathrm{C} / \mathrm{m}^{2}\right)$ & 11.6 & 0 \\
$e_{31}\left(\mathrm{C} / \mathrm{m}^{2}\right)$ & -4.4 & 0 \\
$e_{33}\left(\mathrm{C} / \mathrm{m}^{2}\right)$ & 18.6 & 0 \\
$\kappa_{11}\left(10^{-9} \mathrm{C}^{2} / \mathrm{N} \mathrm{m}^{2}\right)$ & 11.2 & 0.08 \\
$\kappa_{33}\left(10^{-9} \mathrm{C}^{2} / \mathrm{N} \mathrm{m}^{2}\right)$ & 12.6 & 0.093 \\
$q_{15}(\mathrm{~m} / \mathrm{A})$ & 0 & 550 \\
$q_{31}(\mathrm{~m} / \mathrm{A})$ & 0 & 680.3 \\
$q_{33}(\mathrm{~m} / \mathrm{A})$ & 0 & 157 \\
$\mu_{11}\left(10^{-6} \mathrm{~N} \mathrm{~s}^{2} / \mathrm{C}^{2}\right)$ & 5 & 157 \\
$\mu_{33}\left(10^{-6} \mathrm{~N} \mathrm{~s}^{2} / \mathrm{C}^{2}\right)$ & 10 & \\
\hline
\end{tabular}



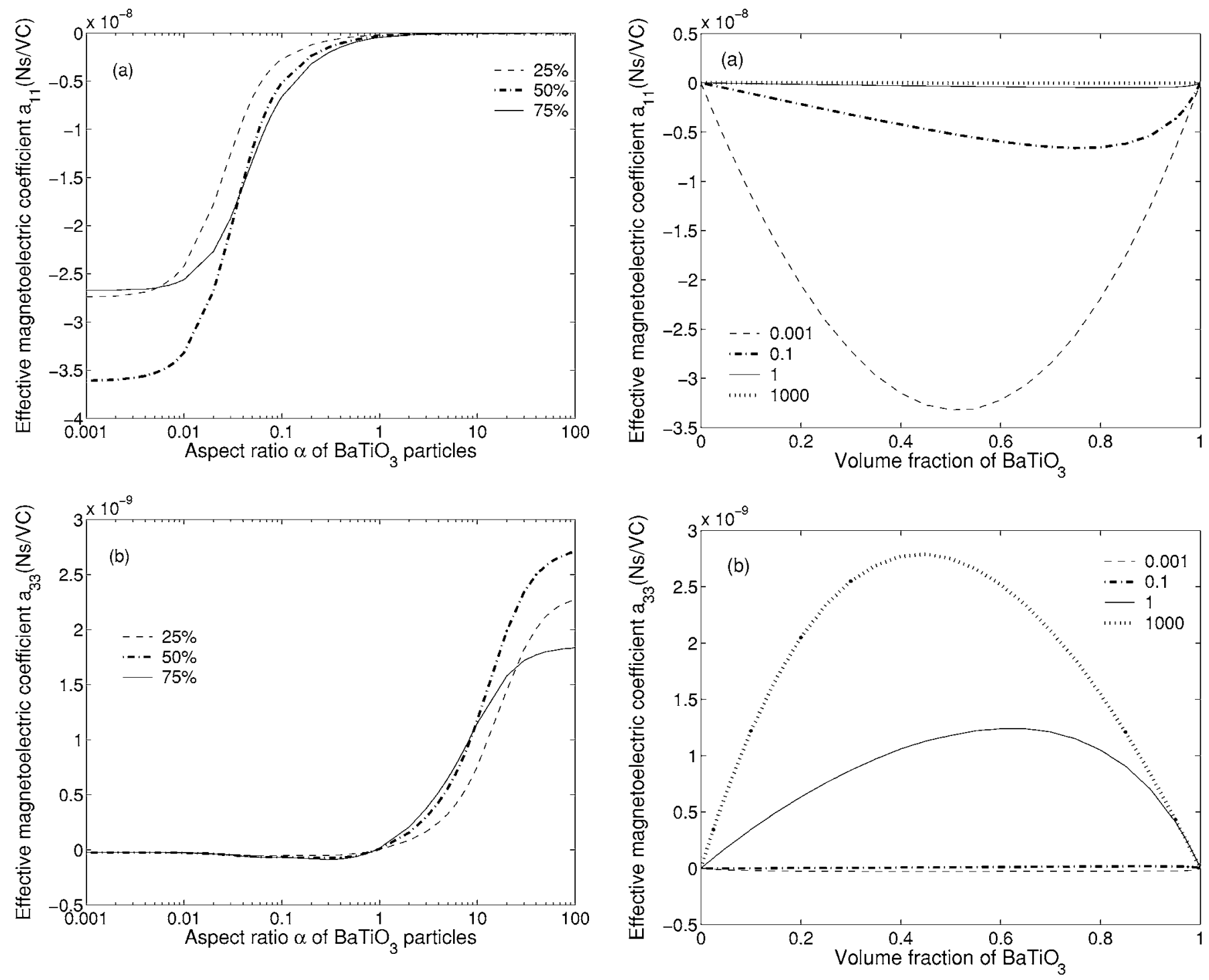

FIG. 1. The effective magnetoelectric coefficients as a function of aspect ratio of aligned $\mathrm{BaTiO}_{3}$ particles at different volume fractions; (a) $a_{11}$ and (b) $a_{33}$.

$\langle H(\theta, \psi, \phi)\rangle=\sum_{i=1}^{I} \sum_{j=1}^{J} \sum_{k=1}^{K} \sin \theta_{i} H\left(\theta_{i}, \psi_{j}, \phi_{k}\right) W\left(\theta_{i}, \psi_{j}, \phi_{k}\right) w_{i j k}$

Such techniques have been used by Rao and Li to study the electrostrictive composites with different orientation distributions of particles. ${ }^{41}$

\section{RESULTS AND DISCUSSIONS}

We have implemented the model by a FORTRAN code, with the algorithm given in the following.

(1) Input the material properties $\mathbf{L}_{1}$ and $\mathbf{L}_{2}$, the volume fractions $f_{1}$ and $f_{2}$, and shape aspect ratio $\alpha$ and texture coefficient $\mu$ for the second phase particle.

(2) For a particle at a particular orientation $(\theta, \psi, \phi)$, transform $\mathbf{L}_{1}$ from global coordinate to local coordinate.

(3) Compute the Eshelby tensor $\mathbf{S}(\theta, \psi, \phi)$ in local coordinate system using $\mathbf{L}_{1}$ and the aspect ratios.

FIG. 2. The effective magnetoelectric coefficients as a function of volume fraction of aligned $\mathrm{BaTiO}_{3}$ particles at different shapes; (a) $a_{11}$ and (b) $a_{33}$.

(4) Evaluate the dilute concentration factor $\mathbf{A}_{2}^{\operatorname{dil}}(\theta, \psi, \phi)$ in local coordinate using Eq. (19), and evaluate $\mathbf{L}_{2}(\theta, \psi, \phi) \mathbf{A}_{2}^{\text {dil }}(\theta, \psi, \phi)$ in local coordinate.

(5) Transform $\mathbf{A}_{2}^{\operatorname{dil}}(\theta, \psi, \phi)$ and $\mathbf{L}_{2}(\theta, \psi, \phi) \mathbf{A}_{2}^{\operatorname{dil}}(\theta, \psi, \phi)$ to global coordinate system.

(6) Average and normalize $\mathbf{A}_{2}^{\text {dil }}(\theta, \psi, \phi)$ and $\mathbf{L}_{2}(\theta, \psi, \phi) \mathbf{A}_{2}^{\text {dil }}(\theta, \psi, \phi)$ to obtain $\left\langle\mathbf{A}_{2}^{\text {dil }}(\theta, \psi, \phi)\right\rangle$ and $\left\langle\mathbf{L}_{2}(\theta, \psi, \phi) \mathbf{A}_{2}^{\text {dil }}(\theta, \psi, \phi)\right\rangle$.

(7) Evaluate $\mathbf{A}_{2}(\theta, \psi, \phi)$ and $\mathbf{A}_{1}$ using Eqs. (17) and (18).

(8) Finally evaluate the effective constitutive moduli using Eq. (15).

The code has been validated by comparing with previously published results under several special conditions. It is applied to calculate the effective magnetoelectroelastic moduli of a multiferroic composite consisting of $\mathrm{BaTiO}_{3}$ and $\mathrm{CoFe}_{2} \mathrm{O}_{4}$ with different volume fractions, shapes, and texture coefficients of second phase particles. The constitutive moduli of both phases are listed in Table I. ${ }^{42}$ Notice that magnetic permeability $\mu_{11}$ of $\mathrm{CoFe}_{2} \mathrm{O}_{4}$ is taken to be the same as $\mu_{33}$. 

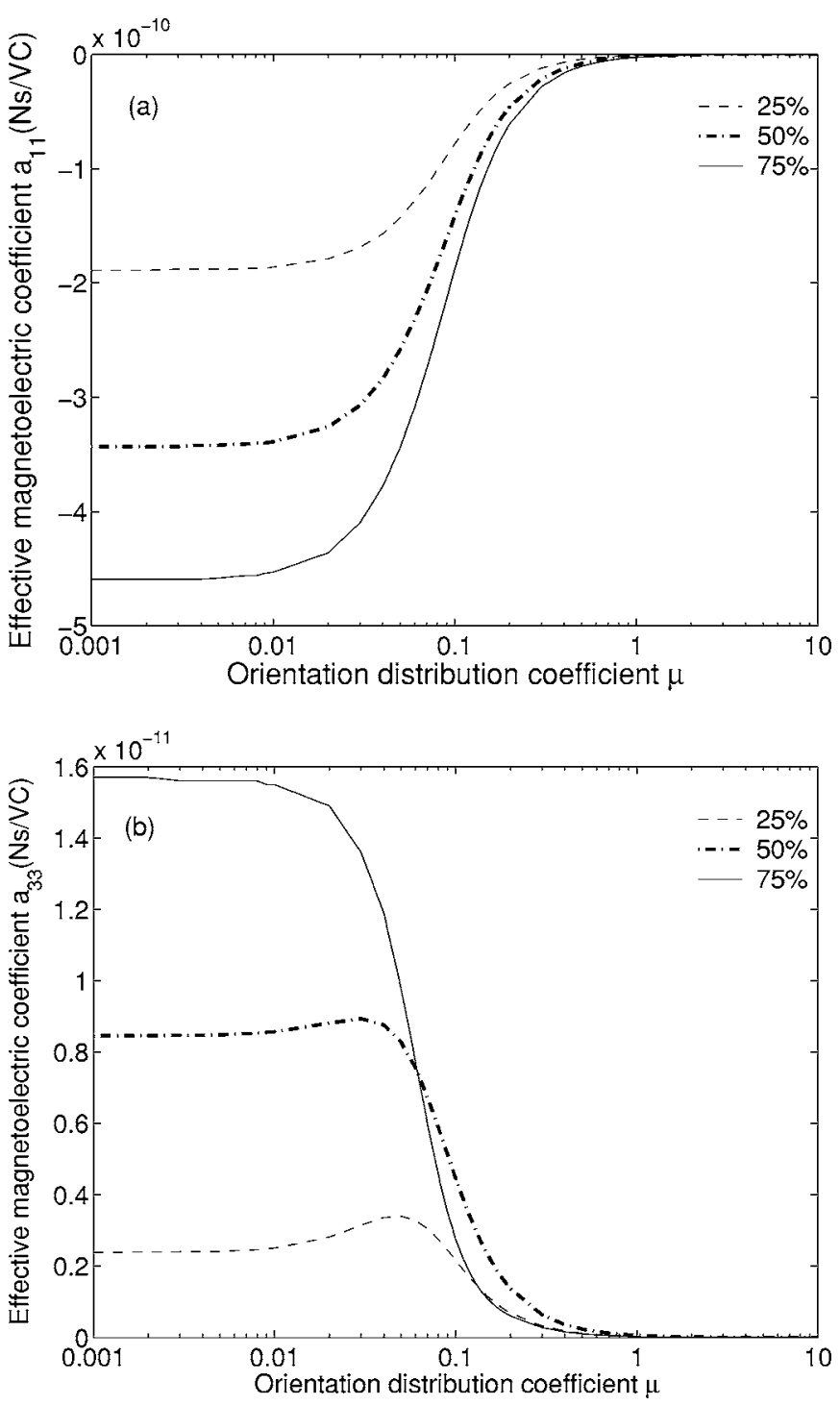

FIG. 3. The effective magnetoelectric coefficients as a function of texture coefficient of spherical $\mathrm{BaTiO}_{3}$ particles at different volume fractions; (a) $a_{11}$ and (b) $a_{33}$.

We first consider the aligned $\mathrm{BaTiO}_{3}$ particles embedded in $\mathrm{CoFe}_{2} \mathrm{O}_{4}$ matrix, with texture coefficient $\mu$ set to be 0.001 . The effective magnetoelectric coefficients as a function of particle aspect ratio at different volume fractions are given in Fig. 1. It is observed that the magnetoelectric coefficient $a_{11}$ decreases with the aspect ratio $\alpha$, while $a_{33}$ increases with $\alpha$. In other words, laminated composites are optimal for $a_{11}$ and fibrous composites are optimal for $a_{33}$. While this has been hypothesized before, we are able to prove it convincingly from our calculations. This is because laminated composites maximize the electroelastic and magnetoelastic interactions between two phases in the $x_{1}$ and $x_{2}$ directions, and fibrous composites maximize those interactions in the $x_{3}$ direction. For the same composites, the effective magnetoelectric composites as function of volume fraction of $\mathrm{BaTiO}_{3}$ at different particle shape aspect ratios are given in Fig. 2, where it is observed that both $a_{11}$ and $a_{33}$ are maximum near $f_{2}=0.5$, again due to the maximized magnetoelectroelastic interactions between piezoelectric and piezomagnetic phases.

We then consider spherical $\mathrm{BaTiO}_{3}$ particles embedded

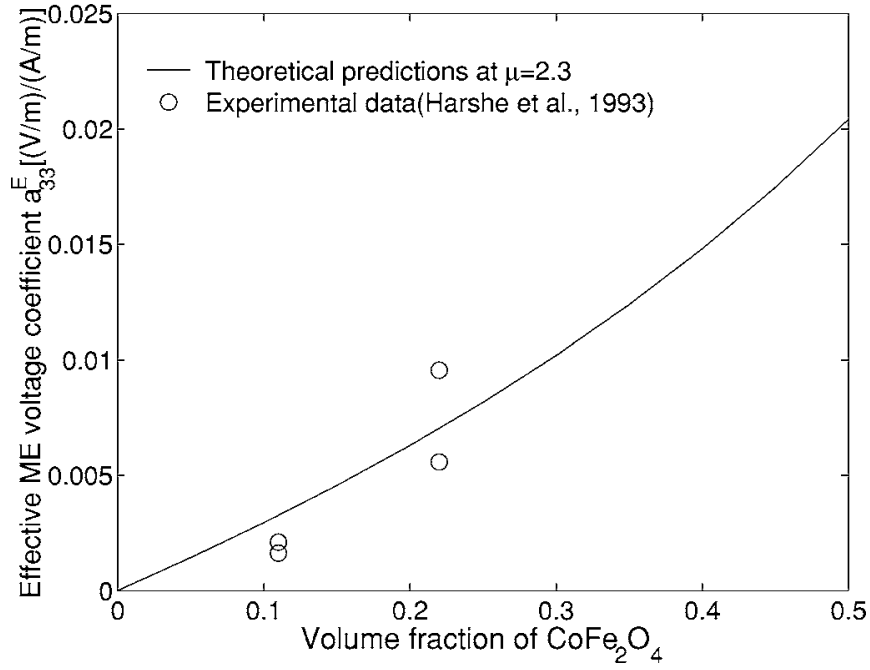

FIG. 4. The effective magnetoelectric voltage coefficient of particulate composites as a function of volume fraction of $\mathrm{CoFe}_{2} \mathrm{O}_{4}$, with the experimental data obtained from Harshe et al. (Ref. 10).

in $\mathrm{CoFe}_{2} \mathrm{O}_{4}$ matrix, with the particle orientation distribution given by Eq. (21). In Fig. 3, we present the effective magnetoelectric coefficients as a function of texture coefficient $\mu$ at different volume fractions, and it is observed that both $a_{11}$ and $a_{33}$ decrease with the texture coefficient $\mu$. In other words, the composites with aligned particles is optimal for magnetoelectric coefficients, which we show convincingly from our calculations. This may also explain the large discrepancy between experimental measurement and previous theoretical predictions for particulate composites, where particles are assumed to be aligned in the calculations which is not very realistic. To demonstrate this, we calculate the magnetoelectric voltage coefficient ${ }^{10} a_{33}^{E}=-a_{33} / \kappa_{33}$ as a function of volume fraction of $\mathrm{CoFe}_{2} \mathrm{O}_{4}$ for a particulate composite consisting of $11 \%$ and $22 \% \mathrm{CoFe}_{2} \mathrm{O}_{4}$ particles embedded in a $\mathrm{BaTiO}_{3}$ matrix; the texture coefficient $\mu$ is set to be 0.23 . Good agreement with the experimental data has been observed, as shown in Fig. 4. It is pointed out that the magnetoelectric voltage coefficient is a more important figure of merit for multiferroic composites, which can be evaluated directly from $a_{i j}$. We also notice that the multiferroic composites are often prepared as piezomagnetic particles embedded in a piezoelectric matrix to take advantages of higher electric resistance of piezoelectric phase, and we expect that the effective behavior of such composites will be qulitativly similar to what we considered here.

Finally, we consider the magnetoelectric coupling factors, defined as

$$
k_{11}^{2}=\frac{a_{11}^{2}}{\kappa_{11} \mu_{11}}, \quad k_{33}^{2}=\frac{a_{33}^{2}}{\kappa_{33} \mu_{33}},
$$

for aligned $\mathrm{BaTiO}_{3}$ particles embedded in $\mathrm{CoFe}_{2} \mathrm{O}_{4}$ matrix, where $\kappa_{11}$ and $\kappa_{33}$ are plotted as function of volume fraction of $\mathrm{BaTiO}_{3}$ particles at different shape aspect ratios, as shown in Fig. 5. It is not surprising to notice that these coupling factors are still very low, and there are much room for improvement. 

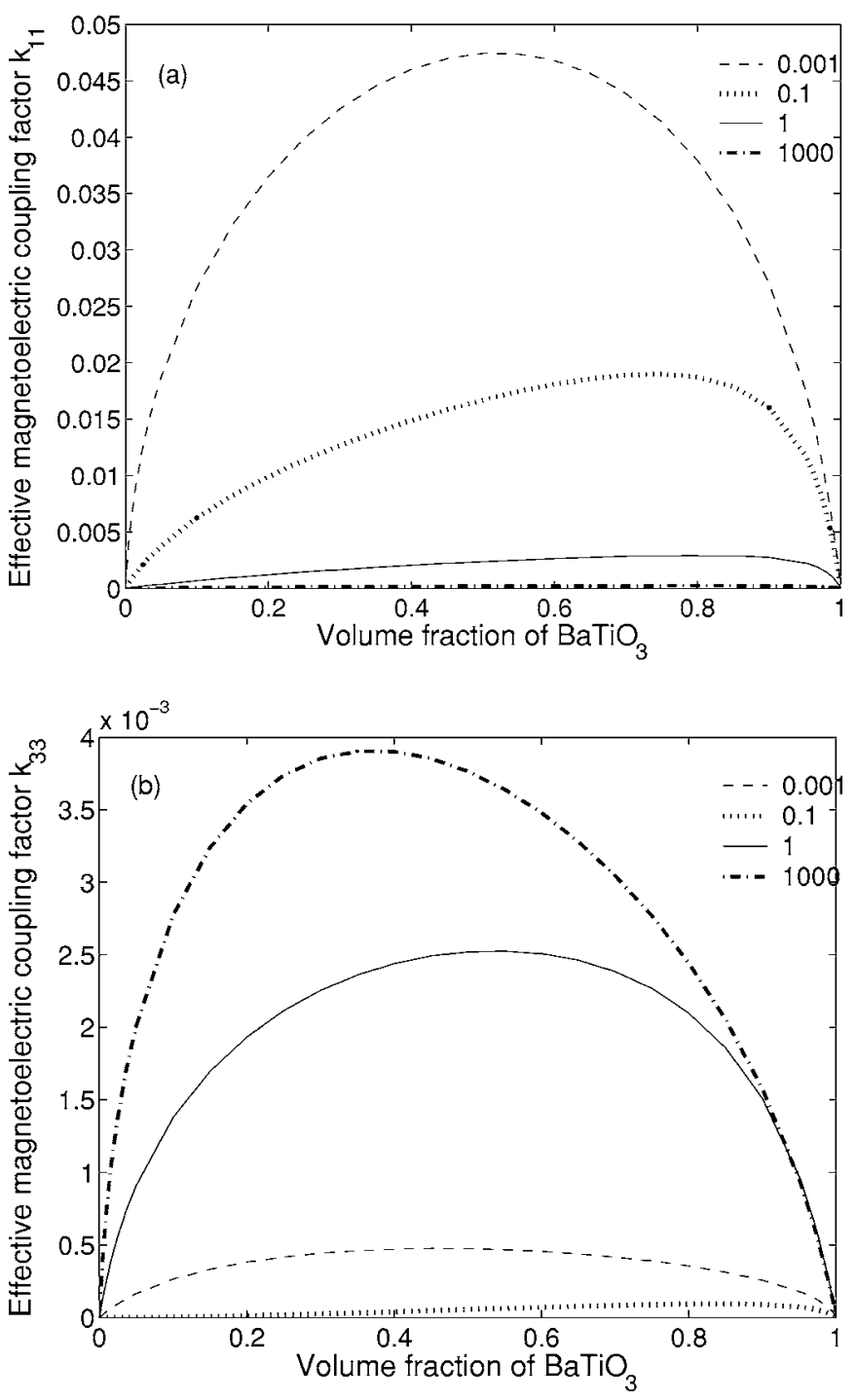

FIG. 5. The effective magnetoelectric coupling factors as a function of volume fraction of $\mathrm{BaTiO}_{3}$ particles having different aspect ratios.

In summary, we developed a mean field Mori-Tanaka model to calculate the effective magnetoelectroelastic moduli of multiferroic composites in terms of the shape aspect ratio, volume fraction, and orientation distribution of second phase particles. Good agreement with experimental data has been observed for particulate composite when the orientation distribution of second phase particles has been considered with appropriate texture coefficient.

\section{ACKNOWLEDGMENTS}

We would like to acknowledge Furong Scholars Programme of Hunan Province, China and National Natural Sci- ence Foundation of China (Approval No. 10572124). We also acknowledge the William Mong Research Fellowship that partially supported one of the author's (J.Y.L.) visit to University of Hong Kong in 2003.

${ }^{1}$ I. E. Dzyaloshinskii, Sov. Phys. JETP 37, 881 (1959).

${ }^{2}$ L. D. Landau and E. M. Lifshitz, Electrodynamics of Continuous Media, 2nd ed. (Pergamon, New York, 1984).

${ }^{3}$ D. N. Astrov, Sov. Phys. JETP 38, 984 (1960).

${ }^{4}$ V. J. Folen, G. T. Rado, and E. W. Stalder, Phys. Rev. Lett. 6, 607 (1961).

${ }^{5}$ G. T. Rado and V. J. Folen, Phys. Rev. Lett. 7, 310 (1961).

${ }^{6}$ Y. Y. Tomashpol'skii, Y. N. Venevtsev, and G. S. Zhdanov, Sov. Phys. JETP 46, 1921 (1964)

${ }^{7}$ S. A. Kizhaev, V. A. Bokov, and O. V. Kachalov, Sov. Phys. Solid State 8, 215 (1966).

${ }^{8}$ A. M. J. G. VanRun, D. R. Terrell, and J. H. Scholing, J. Mater. Sci. 9, 1710 (1974).

${ }^{9}$ L. P. M. Bracke and R. G. VanVliet, Int. J. Electron. 51, 255 (1981).

${ }^{10}$ G. Harshe, J. P. Dougherty, and R. E. Newnham, Int. J. Appl. Electromagn. Mater. 4, 161 (1993).

${ }^{11}$ G. Harshe, J. P. Dougherty, and R. E. Newnham, Int. J. Appl. Electromagn. Mater. 4, 145 (1993)

${ }^{12}$ J. Ryu, A. V. Carazo, K. Uchino, and H. E. Kim, J. Electroceram. 7, 17 (2001).

${ }^{13}$ C. W. Nan, M. Li, X. Q. Feng, and S. W. Yu, Appl. Phys. Lett. 78, 2527 (2001).

${ }^{14}$ C. W. Nan, M. Li, and J. H. Huang, Phys. Rev. B 63, 144415 (2001).

${ }^{15}$ C. W. Nan, Y. H. Lin, and J. H. Huang, Ferroelectrics 280, 319 (2002).

${ }^{16}$ K. Mori and M. Wuttig, Appl. Phys. Lett. 81, 100 (2002).

${ }^{17}$ J. Ryu, S. Priya, and K. Uchino, and H. E. Kim, J. Electroceram. 8, 107 (2002).

${ }^{18}$ C. W. Nan, G. Liu, and Y. Lin, Appl. Phys. Lett. 83, 4366 (2003).

${ }^{19}$ N. Cai, J. Zhai, C. W. Nan, Y. Lin, and Z. Shi, Phys. Rev. B 68, 224103 (2003).

${ }^{20}$ K. S. Chang et al., Appl. Phys. Lett. 84, 3091 (2004).

${ }^{21}$ H. Zheng et al., Science 303, 661 (2004).

${ }^{22}$ C. W. Nan, Phys. Rev. B 50, 6082 (1994).

${ }^{23}$ Y. Benveniste, Phys. Rev. B 51, 16424 (1995).

${ }^{24}$ M. Avellaneda and G. Harshe, J. Intell. Mater. Syst. Struct. 5, 501 (1994).

${ }^{25}$ J. Y. Li and M. L. Dunn, J. Intell. Mater. Syst. Struct. 9, 404 (1998).

${ }^{26}$ M. I. Bichurin, I. A. Kornev, V. M. Petrov, A. S. Tatarenko, Y. V. Kiliba, and G. Srinivasan, Phys. Rev. B 68, 054402 (2003).

${ }^{27}$ M. I. Bichurin, V. M. Petrov, and G. Srinivasan, Phys. Rev. B 68, 054402 (2003).

${ }^{28}$ S. Srinivas and J. Y. Li, Acta Mater. 53, 4135 (2005).

${ }^{29}$ Z. Shi, C. W. Nan, J. M. Liu, D. A. Filipov, and M. I. Bichurin, Phys. Rev. B 70, 134417 (2004).

${ }^{30}$ T. Mori and K. Tanaka, Acta Metall. 21, 571 (1973).

${ }^{31}$ G. J. Weng, Int. J. Eng. Sci. 22, 845 (1984).

${ }^{32}$ Y. Benveniste, Mech. Mater. 6, 147 (1987).

${ }^{33}$ D. M. Barnett and J. Lothe, Phys. Status Solidi B 67, 105 (1975).

${ }^{34}$ J. F. Nye, Physical Properties of Crystals (Oxford University Press, Oxford, 1975).

${ }^{35}$ M. Dunn and M. Taya, Int. J. Solids Struct. 30, 161 (1993).

${ }^{36}$ Y. P. Qiu and G. J. Weng, Int. J. Eng. Sci. 28, 1121 (1990).

${ }^{37}$ J. D. Eshelby, Proc. R. Soc. London, Ser. A 241, 376 (1957).

${ }^{38}$ J. Y. Li and M. L. Dunn, Philos. Mag. A 77, 1341 (1998).

${ }^{39}$ J. Y. Li, Int. J. Eng. Sci. 38, 1993 (2000).

${ }^{40}$ R. J. Roe, J. Appl. Phys. 36, 2024 (1965).

${ }^{41}$ N. Rao and J. Y. Li, Int. J. Solids Struct. 41, 2995 (2004).

${ }^{42}$ J. H. Huang and W. S. Kuo, J. Appl. Phys. 81, 1378 (1997). 